Cad. Est. Ling., Campinas, 46(1):5-19, Jan./Jun. 2004

\title{
MARCADOR NEGATIVO FINAL NO PORTUGUÊS BRASILEIRO
}

\author{
HELY D. CABRAL DA FONSECA \\ (UNICAMP)
}

\begin{abstract}
This study analyses the final negative marker in Brazilian Portuguese (BP), trying to find for such a phenomenon an adequate explanation within the theoretical background adopted - the Principle and Parameters Theory and its updated research on negation.
\end{abstract}

Key-words Principles and Parameters (PP); negation; final negative marker.

\section{INTRODUÇÃO}

Este trabalho trata do fenômeno da negação no PB procurando explicar, através da teoria de sintaxe gerativa, a presença do marcador final negativo no português brasileiro ${ }^{1}$. Em Torres Morais (2001) encontramos expresso de forma bastante elegante o fato de que a negação sentencial no português é pré-verbal:

\begin{abstract}
"No português moderno as sentenças negativas exibem como propriedade marcante a negação sentencial ou predicativa expressa com o elemento 'não em posição pré-verbal, independentemente da valência e finitude verbal, tipo de sentença - principal/subordinada - e modalidade frasal: sentença declarativa, interrogativa, imperativa." (Torres Morais, 2001: 2)
\end{abstract}

Esse posicionamento do marcador pré-verbal pode ser verificado no exemplo abaixo:

(1) Não vi o filme.

$(\mathrm{Neg} \mathrm{V})$

A concordância negativa $(\mathrm{CN})$ ao mesmo tempo em que mantém o marcador préverbal no PB, vale-se da presença de uma palavra negativa (palavras-n) do tipo ninguém, nada, nenhum, caso em que o marcador e a palavra-n entram em uma relação de concordância, quando de dois itens negativos apenas uma negação se obtém. Vejamos o posicionamento de Negrão et alli (1999) sobre a CN:

“... Os fatos da distribuição dos itens negativos nada, nenhum, ninguém, nunca, jamais, revelam que, se dois destes constituintes negativos aparecem na sentença ou se coocorrem com o operador negativo não, nos contextos relevantes, eles não cancelam um ao outro, mas continuam, em conjunto, a expressar uma negação única”.

\footnotetext{
${ }^{1}$ No anexo 1 o leitor encontrará uma lista das abreviaturas utilizadas nesse trabalho.
} 
FONSECA - Marcador negativo final...

(55) mas até hoje ninguém descobriu nada ...

Este fenômeno, também encontrado em várias línguas românicas, entre elas, o italiano, francês e espanhol, tem sido denominado na literatura recente de concordância negativa..." (Negrão et alli, 1999:13).

Os exemplos abaixo são casos de $\mathrm{CN}$ no $\mathrm{PB}$ :

(2) Não vi nada.

(3) Não vi ninguém.

(4) Não fez nenhum esforço.

Neg V palavra-n

Os exemplos em (5) serão alvo de considerações na seção 6 deste trabalho.

(5) a. Não vi não.

b. Vi não.

c. Não vi nada não.

d. Vi nada não.

\section{O PROBLEMA}

Encontramos, nos exemplos a seguir, uma estrutura [V + palavra-n], doravante [V NEG], que é diferente da ordem canônica esperada em construções de CN do PB:

(6) Tem ninguém no chat ... (página da Internet/Brasnet, 3.10.00) ' $n$ ão tem ninguém no chat'

(7) Experimente usar nada. (revista Caras, $n^{\circ} 44,3.11 .00$ )

'experimente não usar nada'

(8) ... jogar futebol ou fazer nada. (Folha de São Paulo, 03.00) '... ou não fazer nada'.

(9) Pratico nenhum esporte. (Projeto Nurc/RF-D2, 158:48) 'Não pratico nenhum esporte'

(10) Eles estão dizendo que sobrou nada . (Hernandes/Galvão/99) 'que não sobrou nada'

Nos exemplos de (6 a 10) a inexistência do não pré-verbal contraria a classificação do português como língua de negação pré-verbal. Considerando que a estrutura [V NEG] está começando a aparecer em registros escritos no PB, e considerando, também, que as mudanças ocorrem primeiramente na fala, para mais tarde surgirem na escrita, podemos aventar a possibilidade de estarmos registrando um processo de mudança lingüística. Procurando determinar a época do surgimento dessa mudança é que fomos consultar dados do Português Antigo.

A estrutura (V NEG) de (5b) vem sendo considerada como de uso comum no nordeste do Brasil (Mioto, 1991, Furtado da Cunha, 1996). No entanto, nosso estudo atesta a presença dessa estrutura em documentos escritos em outras regiões do Brasil. 


\section{CORPORA}

Lembramos que esse trabalho tem sua base nos estudos de sintaxe da teoria gerativa, e que estamos, portanto, verificando ocorrências de fatos lingüísticos, não sendo relevante o cálculo de percentuais desses fatos.

Os dados de língua oral foram extraídos do projeto Nurc e os de língua escrita foram extraídos do jornal A Folha de São Paulo (março - julho/2000), trechos de textos do 'chat' da internet (ano 2000), revistas brasileiras Cláudia, Caras, e Veja no período de julho a dez/2000 e registros do projeto Português Fundamental*, publicado pela Universidade de Lisboa (1987). Em um segundo momento, após termos constatado a existência do fenômeno na escrita, com o objetivo de, diacronicamente, tentar determinar o surgimento da estrutura estudada, consultamos dados de língua escrita do português antigo (PA): textos do corpus Tycho Brahe (Usp/Unicamp)**, do projeto Prohpor (UFBa) e de jornais brasileiros antigos $* * *$. Não estamos quantificando os dados pelo fato de serem as ocorrências em número ínfimo, que seriam desprezados por programas de análise de dados como o Varbrul, por exemplo. ${ }^{2}$

\section{AS PERGUNTAS}

Face à estrutura [V NEG] encontrada nos exemplos 6 a 10 acima, podemos formular algumas indagações com relação à estrutura observada:

\subsection{O fenômeno estudado ocorre em outras línguas? 4.2. Como explicar a estrutura [V NEG] no PB?}

\section{QUADRO TEÓRICO}

A Teoria Gerativa (Chomsky, 1965) vem propondo análises para o fenômeno da negação. O Modelo Princípios e Parâmetros (PP) (Chomsky, 1981) apresenta dois momentos em seu desenvolvimento: 1) Regência e Ligação; e 2) Minimalismo (Chomsky,

\footnotetext{
* Os dados do PE estão no volume II, da série Português Fundamental, do Tomo primeiro. É uma publicação do Centro de Lingüística da Universidade de Lisboa, 1987.

** $\mathrm{O}$ corpus Tycho Brahe está disponível na rede em http://www.ime.usp.br/ thycho/corpus/acess.html e faz parte do projeto "Padrões rítmicos, fixação de parâmetros e mudança linguiística", coordenado pela Profa. Charlotte Galves, Unicamp.

*** Agradecemos à Profa. Ilza Ribeiro por ter possibilitado nosso acesso aos dados do PA.

${ }^{2}$ Para saber sobre a quantificação da negação final no $\mathrm{PB}$, remetemos nosso leitor para o trabalho de Furtado da Cunha (1999). Para saber sobre os tipos de negação no PB remetemos nosso leitor para Mioto (2001). Para informar-se sobre a história da negação na língua portuguesa remetemos nosso leitor para Torres Morais (2001).
} 
FONSECA - Marcador negativo final...

1993). As etiquetas que a Gramática Gerativa usa para as categorias abstratas serão mantidas em inglês nas representações e nas árvores (ver anexo 1).

Alguns dos autores gerativistas, a exemplo de Pollock (1989), Zanuttini (1997), Ouhala (1990), apontam para explicações sobre negação nas línguas, porém não dão conta da presença de um marcador negativo final como no caso do PB. No entanto, partiremos do estudo desses autores para chegarmos à nossa proposta.

Examinando os resultados de pesquisas feitas sobre a negação encontramos parte das respostas relacionadas aos nossos questionamentos. Comecemos pelo estudo da categoria funcional NegP (Negation Phrase).

\section{1 - O núcleo funcional Neg P}

Tomando o modelo de $\mathrm{PP}$ podemos verificar que os autores divergem quanto à posição de NegP. Antes de Pollock, na teoria de PP, INFL era composto de dois conjuntos de traços: Aspect /Agr e Tense (Chomsky, 1981).

Pollock (1989) propõe que sejam esses conjuntos de traços transformados em categorias, AGR e T, tendo como consequêencia um aumento de posições disponíveis no francês e no inglês e, por extensão, a todas as línguas. Por esse raciocínio, os nódulos AGR e T passam a ter duas posições disponíveis em vez de uma. Pollock afirma a existência da categoria funcional Neg projetando NegP, que passa ocupar a posição entre AgrP e TP.

$\mathrm{O}$ autor explora a idéia de que o marcador negativo do francês $n e$ projeta uma categoria de acordo com a arquitetura da teoria X-barra. Enquanto o núcleo de NegP em francês é realizado como $n e$, a posição de especificador aloja o marcador negativo pas. As três categorias funcionais são ordenadas com TP acima, NegP na posição intermediária e AgrP abaixo, como mostra o diagrama em (11):

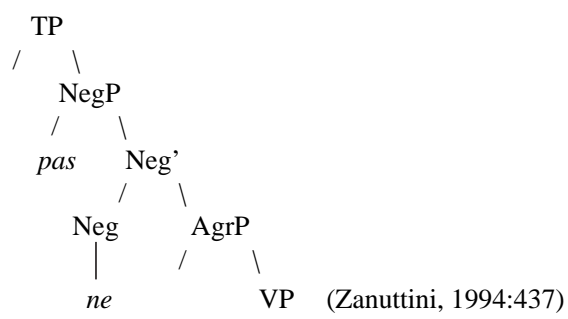

Para explicar a ordem de palavras na superfície, em que ne sempre precede o verbo, Pollock sugere que, pelas suas propriedades de clítico, $n e$ se junte ao verbo, movendo-se junto com o verbo finito para $\mathrm{T}^{\circ}$.

Adotamos essa mesma análise quanto à idéia de que o marcador negativo, no PB, projeta uma categoria $\mathrm{NegP}$, cujo núcleo é realizado pelo marcador não, ocupando a posição de especificador dessa categoria as palavras-n. Pelas propriedades de clítico, num $+V$, movese junto com o verbo finito para $\mathrm{T}^{\circ}$.

Na linha de Figueiredo Silva (1996) estamos assumindo que o item não pré-verbal do PB pode ser clítico: 
Aqui defenderemos a hipótese segundo a qual o PB dispõe de um não clítico que acompanha o verbo, elemento que não deve confundido com o não que pode aparecer isolado. “...O argumento principal é de ordem fonológica: ... não pode ser pronunciado /num/ sem que isso implique degradação ou melhora na aceitabilidade dos exemplos."

(Figueiredo Silva, 1996:56)

Vamos admitir que o não clítico, pode, em contextos apropriados de $\mathrm{CN}$, como nos exemplos de (6 a 10) sofrer processo de apagamento, como aconteceu com o 'ne' do francês.

Em francês moderno, ne não pode negar uma sentença, embora haja casos residuais de ne como operador de negação, ne co-ocorre com outro elemento negativo, por exemplo pas. A maneira de ver ne e pas como núcleo e especificador de uma mesma projeção funcional oferece a vantagem de capturar o fato de que, juntos, eles expressam somente uma situação de negação: um caso de $\mathrm{CN}$ similar ao que ocorre no PB.

(12) Je ne vais pas. ( francês padrão)

Neg V NEG

(13) Je vais pas. (francês coloquial)

V NEG

Zanuttini (1994) propõe manter a idéia das propostas de Pollock (1989) e Belletti (1990), ou seja: que nas línguas Românicas os marcadores negativos podem ser vistos como projetados em NegP e que as diferenças superficiais são resultados de movimentos. Ambos os marcadores pré e pós-verbal, nessa proposta, são gerados em alguma projeção funcional mais baixa que aquela que é o local de pouso do verbo finito.

A proposta de Zanuttini é a seguinte: os marcadores negativos nas línguas romance ou são elementos $\mathrm{X}^{\circ}$ que projetam uma categoria funcional NegP, ou são elementos XP que ocorrem no especificador de $\mathrm{NegP}$, com um $\mathrm{Neg}^{\circ}$ vazio. Os diagramas abaixo ilustram esses dois casos; non do italiano é o núcleo de NegP, enquanto nen do piemontês é um XP, que ocorre no especificador de NegP.

(14)

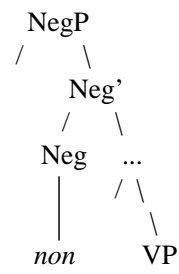

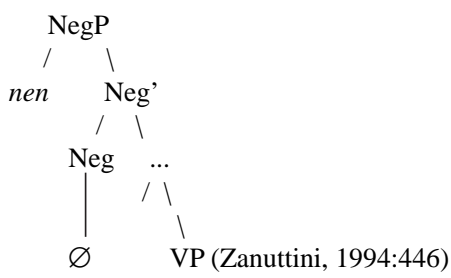

Ao lado da projeção em que os marcadores negativos são gerados na base, uma outra projeção é relevante para a interpretação das sentenças: a projeção PolP (Polarity Phrase), em que esses marcadores são interpretados. Todos os marcadores negativos serão interpretados em PolP. As diferenças existentes entre as línguas com respeito à posição de superfície dos marcadores negativos derivam do fato que, em certas línguas, os traços de PolP devem ser verificados antes do Spell-out, enquanto em outras eles podem ser verificados em LF. Em uma língua como o piemontês, por exemplo, que tem traços 
FONSECA - Marcador negativo final...

negativos fracos, o marcador negativo não se move antes do Spell-out, devido ao princípio de procrastinar, mas subirá em LF. Como se vê a seguir, na árvore de número (16), está a representação proposta por Zanuttini para a negação nas línguas românicas:

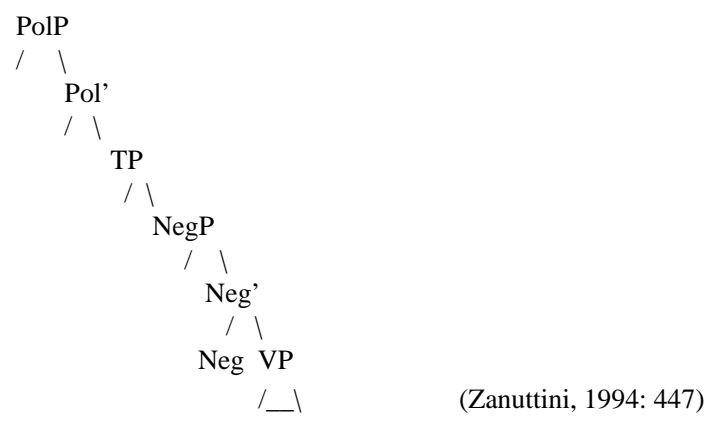

De acordo com Zanuttini as línguas que expressam negação sentencial por meio de um marcador negativo pré-verbal, através de itens que expressam a negação por si sós, são aquelas com traços fortes, que requerem a verificação de traços aconteça antes do Spell-out. Traços fortes forçam o movimento visível. Há duas formas em que os traços de PolP podem ser verificados antes do Spell-out:

(A) movimentando o marcador negativo para o núcleo de PolP, como no caso exemplificado abaixo:

(17) a. Maria non lavora qui. (italiano)(Zanuttini, 1996:16)

b. Maria não trabalha aqui. (português, tradução nossa)

(B) tendo um indefinido negativo no especificador de PolP, de tal forma que a configuração de concordância núcleo/especificador acontece. Este é o caso em que um elemento negativo que é X-max se move para o especificador de PolP, como nos exemplos abaixo:

(18) a. Nessuno há detto niente. (italiano). (Zanuttini, 1994:449)

b. Ninguém disse nada. (português, tradução nossa).

Adotarei aqui a proposta de Zanuttini (1994) no sentido de que NegP pode ser gerado em diferentes posições na base, mas que os traços negativos serão interpretados em PolP, ou na sintaxe aberta, ou em LF

\subsection{NegP, parâmetros e c-seleção}

Existiria um só parâmetro para determinar a negação em todas as línguas? Quando comparamos as conclusões de autores que estudaram a negação, observamos que há concordância em um ponto: NegP é postulado como categoria presente em todas as línguas 
Cadernos de Estudos Lingüísticos 46(1) - Jan./Jun. 2004

examinadas. Porém, quanto a que item lexical pode ocupar o núcleo e o Spec dessa categoria, e quanto a qual categoria $\mathrm{Neg}^{\circ} \mathrm{c}$-seleciona os autores têm propostas diferentes.

Podemos observar, no quadro abaixo, que os pesquisadores concordam quanto ao preenchimento da posição de núcleo e Spec de Neg em francês. Porém discordam quanto a que complemento Neg c-seleciona. Para Pollock (1989) Neg seleciona AgrPs (Agreement Phrase), para Belleti (1990) e Ouhalla (1991) Neg seleciona TP (Tense Phrase), e para Zanuttini (1997) Neg seleciona VP (Verb Phrase).

\begin{tabular}{lclc} 
Francês & Núcleo & Spec & Complemento \\
\hline Pollock (1989) & ne & pas & AgrPS \\
Belleti (1990) & ne & pas & TP \\
Ouhalla (1991) & ne & pas/adv & TP \\
Zanuttini (1994) & ne & pas & Aux/VP
\end{tabular}

Já quando se trata da língua inglesa, os autores têm diferentes posições, sendo que só Zanuttini (1994) vai atribuir status diferente para o $n$ 't e o not. Quanto ao complemento que Neg c-seleciona Ouhalla (1991) e Zanuttini (1994) concordam em parte, como mostra o seguinte levantamento:

\begin{tabular}{lllc} 
Inglês & Núcleo & Spec & Complemento \\
\hline Pollock (1989) & vazio & not & AgrPs \\
Ouhalla (1991) & non & vazio & AgrP/TP \\
Zanuttini (1994) & n't & not & TP/VP
\end{tabular}

Com relação à língua italiana, vemos que as autoras estão de pleno acordo sobre non ocupar o núcleo de NegP, no Spec vai um advérbio, e Neg toma TP como seu complemento.

\begin{tabular}{lllc} 
Italiano & núcleo & Spec & Complemento \\
\hline Belleti (1990) & non & adv & TP \\
Zanuttini (1994) & non & adv & TP
\end{tabular}

Contrariamente a Pollock (1989) e Belletti (1990), que não mencionam que parâmetro estaria envolvido na questão da negação, Ouhalla (1989) afirma que haverá variação paramétrica a depender do tipo de operador que as línguas têm, se afixal ou não, o que iria determinar qual categoria NegP c-seleciona como complemento. Zanuttini (1996) vai optar pelos dois tipos de NegP, NegP1 para as línguas que apresentam negação sentencial préverbal e NegP2 para as línguas que apresentam negação pós-verbal. Nesse ponto, se aproxima de Ouhalla, porque na verdade a autora está postulando diferentes parâmetros para diferentes casos, embora ela não mencione a variação paramétrica, está claro que dois NegPs atestam essa variação.

Assim, percebemos que, em relação aos parâmetros não há uma posição uniforme dos autores. Ouhalla (1991) afirma que diferentes estruturas na sintaxe visível da negação revelam variação paramétrica entre as línguas. No caso do PB, classificado como língua 
FONSECA - Marcador negativo final...

de negação pré-verbal, mas que também apresenta um marcador negativo em posição final, parece haver uma variação paramétrica tal como previsto por Ouhalla.

\subsection{A negação no português brasileiro}

Mioto (1991), na sua tese sobre a negação, apresenta a seguinte representação para o Português Brasileiro (PB):

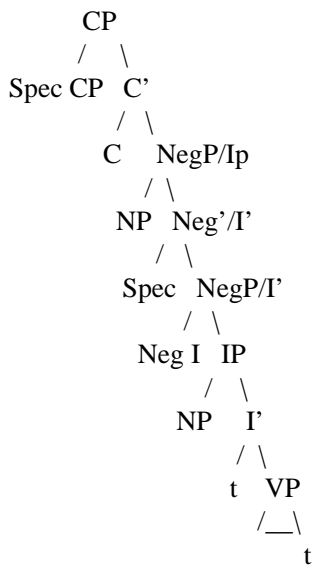

(19) João não visitou a Bahia.
(Mioto, 1991)

Podemos observar que Mioto (1991) propôs um núcleo complexo NegP/IP para a representação da negação em português. Ocorrências do tipo (20) abaixo foram mencionadas por Mioto como negação de "reforço"; do tipo (21) como resposta negativa a perguntas; e (22) como uso regional do nordeste brasileiro.

(20) Ela não veio não. (Neg V NEG)

(21) F1: Ele veio? F2: Veio não. (V NEG)

(22) F1: Você não sabia que eu vinha? F2: Sabia não. (V NEG)

Examinaremos, a seguir, nossas hipóteses para explicar a estrutura (V NEG) com base no quadro teórico adotado.

\section{HIPÓTESES}

6.1. Num é similar ao ne do francês

Retomando a proposta que Pollock (1989) fez para o francês, podemos pensar que algo similar ocorre com o não do PB, que sendo clítico, se afixa ao verbo e se movimenta 
Cadernos de Estudos Lingüísticos 46(1) - Jan./Jun. 2004

da mesma forma que $n e$ do francês para a posição de núcleo de TP, resultando na fórmula [Neg V]. Voltando à analise do $\mathrm{PB}$, o não/num do $\mathrm{PB}$ se cliticiza ao $\mathrm{V}$ e se move para $\mathrm{T}$. Tal como o ne do francês, o item não/num vai sofrer um apagamento em PF mas em LF o núcleo, contendo os traços do operador de negação, continua a existir forçando a subida de TP para a verificação de traços em PolP. Entendemos que dessa forma estamos respondendo à nossa pergunta 4.2 (como explicar a estrutura [V NEG] no PB).

Por outro lado, como resposta à pergunta de número 4.1 formulada anteriormente (o fenômeno estudado ocorre em outras línguas?), vamos dizer que sim, o fenômeno acontece na língua francesa.

Devemos observar, no entanto, que as duas línguas têm diferenças, enquanto o ne do francês perdeu sua capacidade de operador de negação, o seu correspondente não do PB continua podendo negar uma sentença, ainda que haja autores que afirmem o contrário. Martins E. (1997), por exemplo, estudando o português falado no Brasil em um dialeto de Minas Gerais, afirma que:

“...that pre'verbal 'não', which is fully pronounced when used to negate a sentence alone, is only a remnant of the pre-verbal 'não' used in earlier stages of Brazilian Portuguese and which is already used in formal and written language."

(Martins, E., 1997:24)

e:

"the behavior of the phonetically reduced não [num] in spoken Portuguese shows that it has become a negative marker that cannot negate the sentence alone."

(Martins E, 1997:28)

Os argumentos de Martins E. (1997:28) seguem a linha de trabalho anterior de Martins publicado em 1994 para a existência de $\Sigma$ P , com a diferença que Martins E.(1997) propõe mudança paramétrica dos valores de $\Sigma \mathrm{P}$ de forte para fraco no PB moderno. Essa mudança tem como consequiência a dispensa da subida para Sigma, na sintaxe aberta, dos traços negativos para verificação tanto do marcador negativo como do verbo , no caso de sentenças afirmativas.

As observações de Martins E. (1997), igualam o não do PB ao ne do francês quanto à capacidade de negar uma sentença. Essa proposta é diferente da nossa, que considera a presença de traços de um operador de negação em LF, que atua na verificação de traços em PolP.

\subsection{Hipótese 2 - palavras-n $\rightarrow$ operadores de negação}

Já comparamos o num do português com o ne do francês. Notamos que os casos de apagamento do não pré-verbal no PB ocorre na presença das palavras-n nos nossos exemplos (6 a 10). Podemos pensar em uma outra possibilidade, a de que as palavras-n pós-verbais estejam se tornando operadores de negação no PB. Essa possibilidade existe, uma vez que algo similar aconteceu com o pas do francês, além de haver outros casos semelhantes registrados na história das línguas (cf. Martins, 1997, e Roberts \& Roussou, 1999). 
FONSECA - Marcador negativo final...

O ciclo de Jespersen (1917) ilustra bem a questão da queda dos operadores de negação, do surgimento de palavras-n e de minimizadores da negação como novos operadores. O autor, através de um estudo detalhado sobre a evolução da negação, demonstrou que em diferentes momentos das línguas os operadores de negação e as palavras-n ocupam diferentes posições na estrutura frasal, orbitando sempre próximo ao verbo, delineando um ciclo: antes do verbo, antes e depois do verbo numa ação de reforço e depois do verbo. Jespersen mostrou o ciclo completo para o latim, o francês , o inglês e o alemão. Abaixo os exemplos citados pelo autor para o francês:

(23) jeo ne dis(francês antigo, com negação pré-verbal)

(24) je ne dis pas(francês moderno, com negação reforçada)

(25) je dis pas(francês coloquial, com negação pós-verbal)

A constatação de Jespersen se sustenta para o PB, uma vez que temos a negação préverbal exemplificada em (1); a negação, denominada por ele como 'de reforço' como exemplificado em (2, 3 e 4), ou CN na atualidade; e a negação pós-verbal nos exemplos (6 a 10).

Observamos que no PB as três fases do ciclo estão presentes, o que nos leva a pensar na possibilidade de que as línguas possam, de fato, apresentar o ciclo completo concomitantemente, podendo um tipo de negação prevalecer sobre o outro, tendo o uso mais freqüente e mais dominante de um sobre o outro. Mas esse é um assunto para pesquisa futura.

Dentro do modelo adotado, passamos agora para a nossa proposta para dar conta da ocorrência da negação pós-verbal.

\subsection{O marcador negativo final do PB, fortíssimo}

Nos exemplos abaixo notamos a presença de mais um não pós-verbal na estrutura sentencial, provocando a impressão de haver um aumento de itens da negação.

(26) Não vi nada não.

(27) Vi nada não.

Como explicar a presença de um item lexical final que parece aumentar a negação no BP? Acreditamos que o não final nos exemplos 26 e 27 é de natureza diferente do não préverbal clítico, por marcar fronteira de sentença, por ter um contorno entoacional descrescente de final de sentença no PB, ao passo que o não clítico não denota fronteira, admite um outro clítico à sua direita (Ex. não me viu) e tem contorno entoacional crescente, no sentido de que a altura máxima será atingida no verbo.

A presença de um $n a \tilde{o}$, em posição final na sentença, impede a ocorrência de qualquer outro item lexical à sua direita. Qualquer acréscimo à direita do não pós-verbal torna as sentenças agramaticais. Vejamos: 
(20 a) Ela não veio não (*nada).

(21 a) F1: Ele veio? F2: Veio não (* ninguém).

(22 a) F1: Você não sabia que eu vinha? F2: Sabia não (*não).

No entanto, os exemplos modificados (20a, 21a e 22a), acima retomados, podem ser aceitos se usarmos, após o não final, o recurso de uma pausa na fala, ou uma vírgula na escrita, ambas marcações de fronteira sentencial. Fato que corrobora nossa afirmação quanto à posição final do item.

Vamos nos referir ao num clítico, fraco, que sofre apagamento como não ${ }_{1}$ e ao não final, fortíssimo, como $n \tilde{a} o_{2}$. Podemos dizer que o PB distingue as duas funções diferentes do $n \tilde{a} o_{1}$ e do $n \tilde{a} o_{2}$ por suas posições estruturais diferentes.

Estamos adotando o Modelo Minimalista (Chomsky, 1993). Na numeração teremos dois itens ' $n \tilde{a} o$ '. O não, contém, entre outros, um traço forte, uma vez que a teoria propõe a existência de traços fortes e fracos. Quanto ao operador de negação o PB é considerado uma língua de traços fortes (Zanuttini, 1994).

Através das operações de 'mover' e 'concatenar' teremos a sentença (28a), abaixo representada, que não está completa, por restar ainda em nossa numeração os traços formais de PolP e do $n \tilde{a} \mathrm{o}_{2}$. Lembramos que nessa fase o $\mathrm{V}$ se desloca para $\mathrm{T}$ para verificar seu traço de tempo. Quando o V faz essa passagem o não ${ }_{1}$ se cliticiza ao V. Importante notar que para uma sentença estar completa, toda a numeração deverá ser zerada.

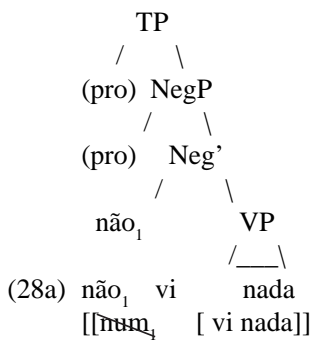

No exemplo $\left(28^{\mathrm{a}}\right)$ temos uma sentença em formação, representada por um TP expelido. Estamos adotando a teoria de múltiplo Spell-out t $^{3}$ de Uriagereka (1999). Na numeração temos ainda os traços de PolP e do não $o_{2}$. O TP, que contém um NegP em cujo núcleo o não ${ }_{1}$ projeta-se, precisa ainda verificar seus traços de polaridade negativa na categoria funcional PolpP, operação que será feita na sintaxe aberta. De acordo com o modelo que estamos seguindo traços fortes motivam movimento na sintaxe aberta. Abaixo a representação arbórea até esse ponto da derivação, em (28b).

\footnotetext{
${ }^{3}$ Spell-out: operação que faz a ligação com o componente PF (Chomsky (1995:189; tradução nossa).
} 
FONSECA - Marcador negativo final...

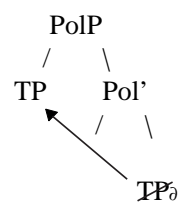

(28b) [[Não ${ }_{1}$ vi nada] [não vi nada]

Enquanto TP é atraído para o Spec (Specifier) de PolP para que haja a verificação de seus traços de polaridade negativa em PolP, os traços do não ${ }_{2}$ projetam-se diretamente do léxico no núcleo de PolP. Temos nessa fase outro spell-out e a sentença é perfeita.

Estamos propondo a existência de um não fortíssimo no PB, o não $o_{2}$, homófono, que tem traços diferentes do $n \tilde{a} o_{1}$ que é átono, reduzido foneticamente e que pode sofrer apagamento. $\mathrm{O}$ não ${ }_{2}$ é tônico, não é clítico, possui traços fortes que serão verificados no núcleo de PolP.

A representação arbórea para (29) está abaixo:

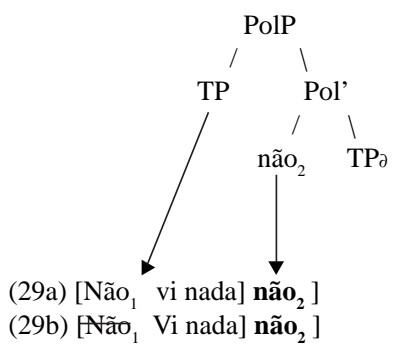

No caso de (29b) a diferença está em que o não é apagado em PF, mas continua atuando em LF, como operador nulo de negação. A pergunta: a negação está aumentando no $\mathrm{PB}$, tem resposta nessa proposta, pois estamos afirmando que o PB tem, definitivamente dois nãos, um que é clítico, que pode sofrer apagamento, como visto nos exemplos de 6 a 10 e $29 \mathrm{~b}$; e um outro que é fortíssimo, que tem um estatuto próprio, isto é, projeta-se diretamente no núcleo de PolP, como nos exemplos de 29 , em posição final da sentença.

Estruturas que, na sintaxe de superfície são diferentes, têm na base, arranjos iguais.

Em (30) e (31) abaixo, seguindo a linha de pensamento que adotamos, podemos afirmar que a negação pós-verbal no PB constitui-se em claro indício de que nos dialetos que admitem essa estrutura o não tem traços fortíssimos, mostrando na sintaxe visível um movimento de TP para Spec de PolP, com o não, ocupando o núcleo de PolP, como explicado acima.

(30) Pegue a criança não.

(31) Não fiz isso nunca não.

O fato de não ser possível colocar nenhum outro item lexical depois do não ${ }_{2}$ pósverbal constitui uma prova de que toda a frase se moveu para a esquerda do núcleo de PolP. 


\section{CONSIDERAÇÕES FINAIS}

Encontramos, dentro do quadro teórico assumido, uma solução satisfatória para explicar as estruturas [V NEG] no PB, apoiando-nos no posicionamento e movimento de TP para Spec de PolP. A estrutura [V NEG] observada nos exemplos de 6 a 10 e de 20 a 22 mostram que há indícios da existência da dualidade do não em PB:

1) um operador negativo, pré-verbal, o $n \tilde{a} o_{l}$, que em casos de $\mathrm{CN}$ pode sofrer apagamento em PF; atuando, porém, em LF como um operador de negação.

2) um operador negativo, pós-verbal, o não ${ }_{2}$, fortíssimo, que se realiza como núcleo de PolP e que ocorre em posição final na sentença.

Por outro lado, considerando-se que a estrutura da negação deve ser sempre marcada por um item lexical, nos casos de apagamento do não ${ }_{1}$ pré-verbal, pensamos que como conseqüência uma das palavras-n no PB poderá vir a ser um operador de negação, tal como ocorreu com o pas do francês. O fato novo é a existência do não $o_{2}$, cuja presença já foi relatada ao longo desse trabalho.

Quanto aos dados consultados do PA e do PE, relatamos que não encontramos a estrutura estudada em tais dados, o que não nos oferece evidência para uma posição clara quanto à existência de [V NEG] no PA e no PE. Não descartamos a possibilidade de que possa haver a estrutura [V NEG] em outros dados a que não tivemos acesso.

\section{REFERÊNCIAS BIBLIOGRÁFICAS}

BELLETI \& Rizzi, L. (1981). "The syntax of ne”. In: The Linguistic Review 1, 117-154. (1990). Generalized Verb Movement. Turin: Rosenberg and Tellier.

CALLOU, D. \& LOPES, C.R.S. (orgs.) (1994). Linguagem falada culta na cidade do Rio de Janeiro: materiais para seu estudo. Rio De Janeiro. Faculdade de Letras/UFRJ. (Volumes: Vol. I - Ef; Vol. II - DID, Vol. III -D2).

CASTILHO, A.T. (1987). Linguagem Falada Culta Na Cidade de São Paulo: Diálogos Entre Dois Informantes. São Paulo: Queiroz / Fapesp.

CHOMSKY, N. (1995). A Minimalist Program. Cambridge, MIT Press. . (1994) . "Bare phrase structure". In: MIT Occasional Papers in Linguistics 5, MIT Press. (1993). "A Minimalist Program for Linguistic Theory". In K. Hale and S. J. Keyser (eds.) The View from Building 20: Essays ins Inguistics in Honor of Sylvain Bromberges. 1/52, Cambridge, MA: MIT Press. (1981). Lectures on Government and Binding. Dordrecht: Foris. (1965). Aspects of the Theory of Syntax. Cambridge, Mass.: MIT Press.

FIGUEIREDO SILVA, M.C. (1996). A posição sujeito no português brasileiro. Editora da Unicamp. Campinas.

JERPERSEN, O. (1917). "Negation in English and other languages". In: Selected writings of Otto Jerpersen. London, G. Allen \& Unwin Ltd.

KAYNE, R. (1994). The Antisymmetry of Syntax. Cambridge: The MIT Press. 
FONSECA - Marcador negativo final...

MARTINS, A. M. (1994). “Aspectos da negação na história das línguas românicas”. In: Actas dos XII encontro nacional da associação portuguesa de lingüística. Editado por Ivo Castro.

MARTINS, E.E. (1997). Sentential Negation in Spoken Brazilian Portuguese. Dissertação de Mestrado. Georgetown University, USA.

MIOTO, C. (1991). Negação sentencial no português brasileiro e teoria da gramática. Tese de Doutorado. Unicamp, Campinas, SP, Brasil. (1993). "Negação sentencial no PB”. In: Boletim da Abralin, 14, pp.105-112. (1998) "Aspectos da sintaxe da negação". In: Anais do XXVII GEL. (1998b) “Tipos de negação”. In: Cadernos de Estudos Lingüísticos, 34, pp.103-117. Campinas, SP, Brasil.

NEGRÃO E. et alli, (1999). "Itens negativos em corpus do português brasileiro" (ms).

OUHALLA, J. (1991). Functional categories and parametric variation. Routledge. London. UK.

POLLOCK, J. (1989). "Verb Movement, Universal Grammar, and the Structure of IP”. In: Linguistic Inquiry, Volume 20, number 3. Summer, pp.365-424.

ROBERTS I. \& ROUSSOU A. (1999). “A formal approach to gramaticalization”. In: Linguistics, Vol. 37-6 (364) p.1011.

SCHWEGLER, A. (1990). Analylicity and syntheticity: A diachronic perspective with special reference to Romance languages. Berlin, Mouton de Greyter.

TORRES MORAIS, M. A ( 2001) . “Aspectos da história da negação no português”. In Mattos \& Silva, R. V. (org.). Para a história do Português Brasileiro. Novos Estudos. Vol. II. Tomo I. SP. Humanitas, pp. 149203)

UkRIAGEREKA, J. (1999). “Multiple Spell-Out”. In: S. D. Epstein \& N. Hornstein (eds). Working Minimalism. Cambridge, Mass: The MIT Press, 251-282.

ZANUTTINI, R. (1997). Negation and clausal Structure. A comparative Study of Romance Languages. Oxford University Press.

. (1994). "Re-examining Negative Clauses". In: Paths towards Universal Grammar. Georgetown University. 
ANEXO 1

\section{Abreviaturas usadas no trabalho}

$\begin{array}{ll}\text { AgrOP } & \text { Agreement phrase } \\ \text { AGRP } & \text { Agreement phrase } \\ \text { CN } & \text { Concordância Negativa } \\ \text { INFL } & \text { Inflection/Phrase } \\ \text { LF } & \text { Logical Form } \\ \text { NEG } & \text { negation/negação } \\ \text { NegP } & \text { Negation phrase } \\ \text { PA } & \text { Português antigo } \\ \text { PB } & \text { Português Brasileiro } \\ \text { PE } & \text { Português Europeu } \\ \text { PF } & \text { Phonological Form } \\ \text { PolpP } & \text { Polarity phrase } \\ \text { PP } & \text { Princípios e Parâmetros } \\ \text { V } & \text { Verbo } \\ \text { VP } & \text { Verb phrase } \\ \text { TP } & \text { Tense phrase }\end{array}$

